\title{
O DEBATE SOBRE A FÁBRICA DE CELULOSE NO RIO URUGUAI: UM CONLFITO ECOLÓGICO DISTRIBUTIVO
}

Mirta Geary

Resumo: Após sete anos de discussão sobre a instalação de um grande projeto de fábrica de celulose às margens do rio Uruguai, o debate deixou de ser um mero conflito diplomático entre os gvernos da Argentina e Uruguai. Neste trabalho, a partir da identificação do caso como um conflito ecológico distributivo, se reconstitui, desde um ponto de vista da ciência política, o cenário no qual vizinhos organizados em assembléias cidadãs, organizações, redes e coalizões ambientalistas, grupos de pesquisadores e técnicos discutiram e geraram estratégias diversas, outorgando visibilidade ao conflito. Se questiona porque, apesar de polêmico empreendimento estar em funcionamento unilateralmente, se continua acionando e argumentando pelos direitos dos cidadãos e dos governos.

Palavras-chave: conflito ecológico distributivo; ações coletivas; acconuntability social; participação cidadã.

\section{THE DEBATE ON THE PULP MI LLS ON THE URUGUAY RIVER: AN ECOLOGI CAL DI STRI BUTI ON CONFLI CT}

Abstract: After seven years of discussion on the installation of a major project of pulp mills on the Uruguay river banks, the debate stopped being a simple diplomatic conflict between the governments of Argentina and Uruguay. On this paper this is identified as an ecological distribution conflict. The scenery in which self-organized neighbors, organizations, networks and environmental coalitions, groups of researchers, technicians and experts discuss and settle different strategies to give visibility to the conflict is reconstructed from a political point of view. This paper also focuses on why, in spite of the start-up in an unilateral way of this polemic project, actions and arguments are still being held for the rights of citizens and governments.

Key Words: ecological distribution conflict, collective action, social accountability, citizen participation.

\section{I ntroducción}

El caso de las plantas de celulosa sobre la margen izquierda del Río Uruguay podría ser leído como un conflicto diplomático, donde, los reclamos entre los gobiernos de Argentina y Uruguay se originan a partir de diferencias producidas en relación al uso del río como un bien público compartido. Sin embargo, a partir del conflicto trasciende que es un problema antiguo mucho más complejo, atravesado por cuestiones de política ambiental, que lleva más de 10 años, donde gobiernos, vecinos organizados, grupos de estudiosos, técnicos y expertos vienen discutiendo y construyendo estrategias con resultados aún inciertos.

El núcleo de la discusión por las papeleras $^{1}$ radicaba en identificar, definir $y$ argumentar la viabilidad ambiental y el riesgo del emprendimiento. Es decir, si el proyecto

\footnotetext{
${ }^{1}$ Nombre a partir del cual inicialmente se identificó el cuestionado proyecto.
} 
ponía en riesgo la salud y las actividades productivas de la población. Hacia el año 2006, al momento de mayor visibilidad del conflicto, se destacan dos cuestiones novedosas: las acciones colectivas de la sociedad civil en defensa de los derechos ciudadanos y ambientales, y el protagonismo del gobierno argentino que, si bien históricamente prestaba poco oído a los grupos ambientalistas, define que el medio ambiente se ha tornado una cuestión de Estado

\section{Antecedentes sobre el proyecto}

Hacia fines del año 2002 se conoce informalmente la posible instalación de una planta industrial de celulosa en las cercanías de la localidad uruguaya de Fray Bentos, frente a la ciudad argentina de Gualeguaychú, centro turístico por excelencia vinculado a la celebración de los carnavales. A partir de esa instancia el gobierno argentino solicita información al uruguayo sobre la entidad del proyecto y una aproximación sobre el posible impacto ambiental de la obra ${ }^{2}$. En una primera instancia los gobiernos discuten el polémico proyecto al interior de las instituciones políticas formales creadas para establecer las reglas de juego: el CARU ${ }^{3}$. Sin embargo, la

\footnotetext{
2 los datos que referencian los antecedentes del contexto de la controversia con el Uruguay provienen del informe oficial del Canciller Taiana a la Presidencia de la Comisión de Relaciones Exteriores y Culto en el Congreso de la Nación (14 de febrero de 2006).

${ }^{3}$ Se trata de la Comisión Administradora del Río Uruguay (CARU). Es el instrumento internacional para la administración de un recurso hídrico compartido. Es creado a partir del Estatuto del Río Uruguay (1975). Esta Comisión es un mecanismo de consulta que pretende guiar la relación y el diálogo entre ambos países para el óptimo y racional aprovechamiento del río, consideran las regulaciones jurídicas en pesca, navegación, subsuelo, etc, donde la preservación de la calidad ambiental del Río Uruguay es el marco que las contiene
}

falta de eficacia de este organismo impidió llegar a acuerdos que permitieran incluir y considerar los argumentos esgrimidos por quienes advertían acerca de los daños ambientales que ocasionaría el emprendimiento. En realidad se trataba de un megaproyecto, donde dos empresas extranjeras son protagonistas: la española Ence, que finalmente sería relocalizada, ${ }^{4}$ y la finlandesa Botnia ${ }^{5}$. Ambas tienen una larga trayectoria en este tipo de industria, pero siendo que, un dato relevante radica en que la primera de ellas había sido condenada en su país de origen por delitos ecológicos, por haber contaminado las aguas marinas y el aire con distintos vertidos industriales en la localidad gallega de Pontevedra.

Las plantas de celulosa han sido históricamente industrias contaminantes en todo el mundo, con factores de riesgo significativos. En América Latina, en países vecinos como Chile y Brasil se registran experiencias concretas de otras comunidades en cuyos territorios se instalaron pasteras. En ambos casos los estudios revelan el alcance de los costos ecológicos y sociales de estos modelos, que se minimizan, o en muchos casos no se consideran, debido a que las empresas logran legitimar la apropiación privada de los bienes ambientales, priorizando la eficiencia económica sobre la equidad social (Leis 2001:137), pero, además, cuentan frecuentemente con un Estado que acompaña políticamente a las empresas, dejando hacer; no controlando ni regulando (Meirelles 2005:30) (Cuenca Berger 2005:36-39). A su vez estos lobbies locales, operan comúnmente como

\footnotetext{
${ }^{4}$ Hacia fines de diciembre de 2006, debido a que su construcción aún no se había iniciado y el gobierno español intercede -ya que operó como mediador en el conflicto- para la reubicación.

${ }^{5}$ Botnia del Grupo finlandés METSA BOTNIA y la empresa Celulosas de M'bopicuá
} 
fragmentadores y destructores de las redes de la sociedad civil .

También se advierte que en otros países, los estudios de los especialistas han orientado las legislaciones, introduciendo nuevas exigencias tecnológicas, donde la calidad ambiental es considerada como bien público, aceptando que debe ser resguardada y regulada por el Estado.

En el caso de España, las consecuencias de la instalación de estas plantas han sido denunciadas por actores locales, semejantes a los que hoy se movilizan y accionan contra el proyecto Botnia. El fallo judicial sobre esta empresa se sostiene a partir de la oposición de los pobladores y expertos científicos que los acompañaron, quienes evaluaron que las consecuencias del funcionamiento de la fábrica originaban una destrucción paulatina de los sistemas naturales, mermando los recursos pesqueros y provocando la contaminación que ha afectado la salud de decenas de miles de personas. Se observa entonces que en este caso las acciones de los grupos organizados han podido incidir en las decisiones políticas del gobierno. La justicia de su país ha considerado los cuestionamientos del impacto ambiental y las leyes se han encargado de arrinconar este tipo de "industria sucia” poniendo límites a la contaminación. ${ }^{6}$ En este sentido debemos tener presente que las decisiones de política ambiental se caracterizan frecuentemente porque, si bien se toman en el presente, tienen impacto futuro (en algunos casos,

6 La expresión de arrinconar a las industrias contaminantes a partir de legislaciones proviene del Informe de Greenpeace, en el marco de la Campaña Tóxicos, enero de 2006, titulado Plantas celulosa sobre el río Uruguay. Nueva amenaza de una industria sucia . Greenpeace.org.ar incluso, suponen efectos irreversibles, tanto en los humanos como en los no humanos), y también por el elevado grado de incertidumbre respecto a sus consecuencias. Martínez Alier (2000:195) considera que los dos problemas están fuertemente ligados, la incertidumbre siempre es, obviamente, sobre el futuro, y es mayor cuanto éste es más lejano

La impugnación al proyecto inconsulto del gobierno uruguayo puede situarse no sólo en referencia a los acuerdos bilaterales de ambos países, sino también en el marco de conocidos compromisos ambientales firmados posteriormente por los gobiernos a nivel mundial ${ }^{7}$, en los cuales se sientan las bases para transformar el modelo de desarrollo bajo un criterio de desarrollo sustentable y donde acordaron que, en los planos locales, éste podría ser viable a partir de agendas ambientales (A21 Locales), como resultado de un debate y negociación entre el gobierno y todos los sectores de la sociedad civil.

En este contexto, la movilización de la sociedad civil contra las pasteras surge en el año 2003, por parte de los grupos ambientalistas uruguayos, quienes alertaron a las organizaciones de Gualeguaychú y comienzan a reunirse como vecinos autoconvocados por la defensa del Río Uruguay (Merlinsky 2008:38), y cuya idea era delinear una estrategia para oponerse a la instalación de las pasteras. Este fenómeno de movilización de la sociedad civil no debe ser pensado como un mero reflejo ante el problema de la instalación de las papeleras, ya que las propuestas de discusión de las A21

\footnotetext{
7 en el marco de la Conferencia de las Naciones Unidas para el Medio Ambiente y Desarrollo, que en el año 1992 asumieron en Río una propuesta de acción para transformar seriamente el modelo de desarrollo económico en el Siglo XXI a través de los Programas Agenda 21 (A21) y A21 Locales.
} 
locales han comenzado a ser motorizadas, mayormente a partir des las organizaciones, a través de formas diversas de participación desde hace más de una década.

En este sentido, se observa que gran parte de las ONGs de los países de América Latina vienen construyendo coaliciones o redes nacionales desde hace bastante tiempo. A menudo su ámbito de interés está referido al monitoreo de los llamados mega proyectos de desarrollo, que generalmente presentan impactos en la biodiversidad y en las propias comunidades locales. Los actores que componen estas redes son diversos y múltiples, algunos permanentes y otros transitorios. Comúnmente lo que proponen estas coaliciones es generar una visión colectiva para la acción conjunta sobre la sustentabilidad en el manejo de recursos, incluyendo la mirada de diferentes sectores y generando estrategias, orientadas a potenciar iniciativas que permitan influir en la discusión sobre la política ambiental. Estas coaliciones cuentan con una masa crítica que permite organizar y coordinar formas de acción colectiva a partir de estrategias consensuadas. La importancia de las mismas también reside en su capacidad para gestionar espacios de participación en foros de discusión internacionales teniendo en cuenta los convenios Internacionales sobre el Ambiente: Diversidad biológica, Cambio Climático, Bosques y Aguas -entre otros-, impulsando procesos de construcción social y políticas sobre procesos de participación ciudadana y fortalecimiento de las organizaciones.

Un mes antes de la asunción del gobierno de Tabaré Vásquez (febrero 2005) el gobierno saliente otorga la autorización ambiental preliminar a la empresa Botnia para su proyecto de planta de pasta, terminal portuaria y zona franca. Es decir, toma una decisión unilateral que no sólo materializaría el inicio de la construcción de las obras de las papeleras, sino que rompe el diálogo y los términos de cooperación, establece reglas propias con efectos distributivos, y reactiva las discusiones en el marco de los diversos grupos y actores que interactúan en el espacio público.

Esta decisión operó como disparador para la visibilidad de las acciones colectivas y la organización de la ciudadanía en reclamo de sus derechos, iniciando formas de accountability.

A fines de abril de 2005 se organiza un "abrazo" de 40 mil personas, que unió las dos orillas del puente que comunica Gualeguaychú con Fray Bentos, en las cercanías del lugar de radicación de las plantas. Para la sociedad civil y las organizaciones ambientalistas de ambos países esa inédita y masiva movilización marca un antes y un después en la discusión de la instalación de las papeleras. ${ }^{8}$

En este contexto los Presidentes Kirchner y Vásquez acuerdan crear una nueva instancia de discusión: el llamado Grupo Técnico de Alto Nivel Argentino-Uruguayo (GTAN) que durante ocho meses (mayo 2005/enero2006) convocaría a expertos, técnicos y especialistas, cuyo conocimiento sería la base común para estudiar y discutir nuevamente las consecuencias de las papeleras en funcionamiento sobre el ecosistema del río Uruguay, evaluando en qué medida el proyecto podría tener efectos ambientales transfronterizos. Aquí el Medio

\footnotetext{
8 El grupo Guayubira (Uruguay) y la Comisión Nacional en Defensa del Agua y la Vida fueron sus organizadores . La movilización sumó representantes del sistema político argentino, simpatizantes del Frente Amplio uruguayo e intelectuales
} 
Ambiente se transforma en una cuestión de Estado (Merlinsky 2008: 42).

Si bien el informe no tendría carácter vinculante, permitiría intercambio de información, un recurso que, en términos de agendas ambientales urbanas fortalece a la sociedad civil en cuanto actor interviniente y es pensada, como la base sobre la cual se construyen relaciones de confianza y cooperación entre gobiernos, ciudadanos y empresas (Araya Dujisin, 2001).

Sin embargo, y pese a esto, en el marco de este escenario, el gobierno entrante uruguayo decide autorizar oficialmente a la empresa Botnia (julio 2005) la concesión para construir y operar una terminal portuaria para actividades específicas de la planta de celulosa. Durante la campaña electoral para la presidencia (2004), Tabaré Vázquez (conjuntamente con su alianza política Encuentro ProgresistaNueva Mayoría-Frente Amplio) había evitado debatir públicamente el proyecto de construcción de las dos plantas de celulosa y a su vez el actual presidente, José Mujica, siendo su ministro de Ganadería, precisó que la forestación degrada los suelos, y sentó posición al manifestarse contrario a subsididar a las multinacionales forestadoras (Zibechi, 2005)

De esta manera, lo que en Fray Bentos comienza como un problema ambiental se transforma en un conflicto.

Las impugnaciones hacia el proyecto se fundaban en los efectos contaminantes que producirían estas plantas en funcionamiento $y$ es por eso que las comunidades locales reclamaban un informe técnico, que diera cuenta de las tecnologías que emplearán las industrias, del impacto preciso que tendrán en los centros urbanos próximos y de la preservación del medio ambiente en el ecosistema del río Uruguay ${ }^{9}$. Es imposible separar el problema del agua en relación a la producción de celulosa y el monocultivo de eucaliptos ${ }^{10}$

\section{El agua, las pasteras y el conflicto de distribución ecológico}

La producción de pasta de celulosa tiene particularidades muy significativas en relación al ecosistema, ya que se basa en un doble consumo intensivo de recursos, tanto de madera como de agua. La madera proviene de las extensas plantaciones de eucaliptos de la zona, donde las mismas empresas son propietarias $^{11}$. El agua en cambio es aparentemente un recurso "sin precio", "sin dueño", un bien común o público, pero sin duda es el factor determinante para comprender porqué estas

\footnotetext{
9 La tecnología radica en lo que se conoce como Proceso Kraft", a través del cual se propone la utilización del dióxido de cloro en el proceso de producción industrial- es intrínsecamente contaminante por lo que requiere la adopción de medidas para prevenir el impacto ambiental que pueden producir los efluentes líquidos, las emisiones gaseosas y los residuos sólidos. En este sentido, al momento del conflicto uno de los principales defectos de la información disponible en los ElA (Estudios de Impacto Ámbiental) de las empresas era en el borrador de ElA Acumulado presentado por la Corporación Financiera Internacional (CFI) donde se advertía la ausencia de medidas concretas y específicas para prevenir la liberación de elementos contaminantes en los efluentes líquidos y las emisiones gaseosas, mitigar el impacto ambiental de los contaminantes que resulten liberados a pesar de esas medidas de prevención, establecer un sistema de gestión ambiental y para el control de las contingencias que puedan suscitarse. (Datos extraídos del Informe del Canciller Argentino Dr. Taiana ante la Comisión de Relaciones Exteriores y Culto en el Congreso Nacional, 14/2/2006)

10 Para este tema ver Meirelles (2005)

${ }^{11}$ Las empresas Botnia y Ence son propietarias de 100.000 ha de tierra cada una, en las que crecen sus eucaliptos y la mayoría de las plantaciones tiene 1.100 árboles por hectárea. Informes pormenorizados acerca del impacto del modelo forestal que va hermanado con la construcción de las papeleras, las tecnologías de las plantas de celulosa y los datos que permiten discutir la apropiación y destrucción de recursos esenciales para generaciones futuras por parte de empresas tanto nacionales como extranjeras pueden ser consultados en los trabajos del Grupo ambientalista “Guayubira" en el sitio www.guayubira.org.uy
} 
industrias buscan instalarse en estas tierras, en la margen misma del río Uruguay y no en otro lugar. El hiper consumo del agua se traduce en cifras impensables, que incluye tanto el proceso de industrialización como el uso intensivo para el monocultivo de eucaliptos $^{12}$. A modo de ejemplo se calculaba que estas fábricas iban a utilizar en un día lo mismo que la ciudad de Fray Bentos en un mes. ${ }^{13}$ (Meirelles p.30 y 32, 33 y 34)(Cuenca Berger 2005:36-37 y 39) (Achkar y otros 2005:55)

La diferencia sustantiva entre un problema y un conflicto ambiental es que en el primer caso no existen acciones por parte de los afectados. El conflicto conlleva acciones de los potencialmente afectados, siendo que el objetivo es evitar un daño ambiental o lograr su reparación"14. En realidad estamos ante lo que la ecología política aborda como conflicto de distribución ecológica, concepto que se refiere a "las asimetrías o desigualdades sociales, espaciales y temporales producidos por los humanos en la utilización de los recursos y servicios ambientales"(M. Alier,1997:216). En el caso de las pasteras esto se traduce en la amenaza de un daño, que algunos, en vías de

12. Tanto geógrafos como naturalistas afirman que el alto consumo de agua en los monocultivos a larga escala se debe a la cortedad de su ciclo de desarrollo (Meirelles 2005:38). La mayoría de las plantaciones tienen 1.100 árboles por hectárea. A su vez se suma al consumo cuantitativo del agua, el uso cualitativo, ya que a menudo los ríos sufren efectos contaminantes debido al uso de fertilizantes químicos y agrotóxicos. (Meirelles, 2005: 38)

${ }^{13}$ Esto remite a la magnitud del megaproyecto, ya que las dos plantas representan el doble de la capacidad de producción actual de pasta de papel que posee Argentina a través de las diez empresas. De modo que esto se traduciría en una concentración de contaminantes frente a las costas de Gualeguaychú, superando, según Greenpeace, todo lo conocido para esta industria en la región. (http://www.greenpeace.org.ar)

14 Observatorio Latinoamericano de Conflictos Ambientales de Chile (1989), citado en Colombo et al, Revista I beroamericana de Economía Ecológica. minimizarlo e impulsar el proyecto sin someterlo a discusión, lo definían como “contaminación controlable" o "niveles de contaminación controlables" y en cambio otros como "irreparable". ${ }^{15 " . ~ P e r o ~ n o ~ t o d o s ~}$ los humanos son igualmente afectados por el uso que la economía hace del ambiente natural. Unos se benefician más que otros, unos sufren más que otros, de ahí los conflictos ecológicos distributivos o conflictos de justicia ambiental." (M. Alier 2006:2). A su vez, el prestigioso economista ecológico sostiene que los actores de estos conflictos usan diversos lenguajes de valoración, que contemplan diferentes tipos de derechos y valores ecológicos, que no pueden expresarse en dinero. ${ }^{16}$

En el conflicto por las pasteras, las acciones de los vecinos organizados de la costa entrerriana -Gualeguaychú, y posteriormente Colón y Concordia comienzan a partir de la falta de cumplimiento de las reglas de juego institucional, del avance material en la construcción de una de las plantas, Botnia, ya que ENCE había desistido y decidido trasladar su emplazamiento

Los vecinos se nuclearon $y$ organizaron bajo la forma de "asambleas ambientales ciudadanas", donde evalúan la información y discuten estrategias de acciones. De aquí surgen los cortes de ruta como una de las formas más visible, polémica y persistente de protesta. Esta medida,

15 el trabajo de Willat (2005) relativiza este argumento a la luz de datos donde analiza la cadena productiva de la celulosa y su relación con las tecnologías en cuestión, en el marco de las obligaciones de contralor por parte del Estado.

${ }^{16}$ Cabe destacar que la Economía Ecológica estudia las relaciones entre al economía y el medio ambiente (lo que incluye el debate sobre la sustentabilidad ecológica de la economía y el debate sobre el valor de los daños ambientales). La Ecología Política estudia los conflictos ecológicos distributivos."(M. Alier op,cit.2) 
aparte de impedir la libre circulación de los puentes internacionales que unen Uruguay y Argentina en plena temporada turística, generando apoyos y rechazos, fueron una estrategia para reclamar y presionar ante los gobiernos local, provincial y nacional. Apuntaban a concientizar a los políticos y a la comunidad en general acerca de la incertidumbre sobre el alcance de los daños ambientales que significaría la instalación del megaproyecto en esa localización.

\section{Accountability social}

La estrategia de los cortes de ruta muestra formas organizadas de acción colectiva, que si bien algunos visualizan como exceso de ambientalismo o fundamentalismo ecológico, pueden, en la práctica, transformarse en modalidades de control social organizado y selectivo, lo que Peruzotti y Smulovitz (2002) definen como accountability social, , o nuevas formas de control de las autoridades políticas. Este concepto intenta captar nuevas formas de participación de ciertos actores sociales, capaces de producir efectos políticos no sólo novedosos, sino que hacedores de influencia en el espacio público. Pueden ser leídos como "mecanismos" que, al apoyarse en el accionar de sectores organizados de la sociedad civil y de los medios de comunicación, aspiran a controlar política y legalmente las acciones de los políticos y burócratas (2002:34). Las iniciativas de accountability social pretenden monitorear el comportamiento de funcionarios públicos, denunciando actos ilegales -como el no cumplimiento del Estatuto del río Uruguay por ejemplo- y pueden también activar la operación de agencias del Estado, como instituciones responsables de controlar a las industrias contaminantes. Este tipo de control pretende a su vez exponer públicamente temas ignorados, pugna para introducirlos en la agenda pública, y generar un debate político, que aporte información y protagonismo a la ciudadanía.

Pero hay algo significativo para el análisis de la sociedad civil y de la opinión pública política, para la comprensión de las estrategias de los actores, y es que, esta complejidad de la comunicación política se define en última instancia en la resonancia y en el asentimiento de un público de legos. Es aquí donde el público de los ciudadanos ha de ser convencido por contribuciones e intervenciones inteligibles que despierten interés para todos a partir de temas que se han percibido como trascendentes. Lo que Habermas (2000) concibe como una autoridad del público, deviene como constituida de la estructura interna del espacio de la opinión pública, que es en el cual los actores pueden presentarse.

En las diferentes perspectivas de las teorías del espacio público, éste puede entenderse como ámbito de expresión pública para participar en deliberaciones o manifestaciones respecto a temas de interés colectivo y también como mecanismo alternativo para la toma de decisiones. El espacio público tiene para Habermas (2000) una función crítica, en la cual la sociedad civil es la encargada de mantener intactas las estructuras comunicativas del espacio de la opinión pública, y hay un público del espacio público, que puede estar en reposo o en movilización. El primero responde a la imagen de la sociología de los medios de comunicación, donde la sociedad civil no podría influir, mientras que el segundo es el que tiene capacidad de influir en el sistema político. Se trata de un público informado y movilizado.

Los reclamos organizados de los vecinos y asambleístas, parecen orientarse 
en esta perspectiva: un público informado y movilizado que aspira a recuperar la confianza social. Y aquí cabe una reflexión. La confianza social en los conflictos ambientales no radicaría sólo en rescatar su importancia como el lubricante de la sociedad y su significación en torno a la credibilidad y legitimidad de las instituciones, sino en enfatizar que también importa también la confianza social que tiene la gente en la acción colectiva, en lo que hace la sociedad civil como tal, ya que, la contracara sería la autodecepción colectiva.

En este contexto de debate polarizado donde es posible identificar actores y discursos, donde se advierte un oído político más dispuesto para escuchar y por tanto a considerar al menos algo de los debates de la sociedad civil en términos de políticas públicas, es aún difícil prever el modo de resolución del conflicto. Una pregunta que merece ser planteada para este conflicto sería bajo qué condiciones las personas miembros de las organizaciones deciden participar y sostener la participación en acciones individuales o colectivas destinadas a impulsar o decidir sobre cuestiones trascendentes de política ambiental, ya instaladas en al agenda pública?.

\section{Consideraciones finales}

Durante los últimos cuatro años la sociedad civil se mantuvo firme en las medidas de fuerza, reclamando el cierre definitivo de la planta, que hace tiempo funciona a toda máquina en Fray Bentos, intentando incidir en el sistema político argentino. Mientras tanto, el gobierno nacional argentino había presentado, en mayo de 2006, una medida cautelar ante la Corte Internacional de Justicia $(\mathrm{CIJ})$, en La
Haya, y el gobierno uruguayo había reclamado por los cortes de ruta ante el sistema de solución de controversias del MERCOSUR ${ }^{17}$. Dos meses después del reclamo, la $\mathrm{ClJ}$ define que la Argentina, de acuerdo a los informes presentados, no ha convencido a la Corte acerca del daño ambiental irreparable y por lo tanto rechazaba el pedido argentino para detener las obras de construcción de las plantas. ${ }^{18} \mathrm{Al}$ mismo tiempo el tribunal manifiesta la necesidad de velar por la protección del Medio Ambiente y recomienda a los dos países iniciar un diálogo de buena fe.

El largo trámite de los procedimientos exigidos por la $\mathrm{ClJ}$ por la presentación caratulada como “Pasteras en el río Uruguay(Argentina vs Uruguay)"demandó varios años y finalmente los gobiernos de Argentina y Uruguay iniciaron las negociaciones a través de los Presidentes Cristina Fernández de Kirchner y J osé Mugica. En junio de 2010 el tribunal falló señalando que ambos países deben controlar que el río en común no sea contaminado, incluyendo las industrias de ambas orillas, un monitoreo de la empresa desde su interior y que los mismos deberían estar a cargo del CARU (Comisión Administradora del Río Uruguay). La asamblea de Gualeguaychú deliberó de manera continua y pronunciada, y, a través de una votación pareja, resolvió levantar el corte de la ruta ${ }^{19}$ de acceso al puente que une las ciudades de Gualeguaychú y Fray Bentos y negociar con el gobierno nacional un monitoreo conjunto a la pastera UPM (ex Botnia).

\footnotetext{
17 Para la presentación ante la Corte Internacional de Justicia se recomienda ver el trabajo de M. Colacrai (2008)

18 La pastera ENCE se relocalizaría 5 meses después del fallo, como ya hemos mencionado

${ }^{19}$ En principio el levantamiento es por 60 días, la decisión fue tomada el 16 de junio de 2010
} 
Este proceder de los vecinos organizados como sociedad civil a través de la Asamblea expresa la importancia de estas formas alternativas para la toma de decisiones, mostrando que las luchas de la ciudadanía extendidas en defensa de la justicia ambiental para proteger los bienes comunes, están dadas y resignificadas a través de experiencias deliberativas (Castro 2008:78). Los vecinos expresaron que el levantamiento del corte estaría condicionado a las negociaciones que ambos gobiernos se den acerca de las modalidades de monitoreo y control sobre el impacto ambiental de las fábricas.

En este sentido se observa que luego de marchas y contramarchas entre los gobiernos, y reuniones de la cancillería argentina con los asambleístas para deliberar acerca del Plan de Control Ambiental del Río Uruguay a presentar, Fernández de Kirchner y Mujica firmaron un acuerdo para el monitoreo conjunto el 28 de julio de 2010. El mismo fue considerado por los medios de comunicación como un paso crucial para la resolución de la crisis y la desactivación del conflicto.

A partir de aquí se abre un nuevo camino, donde el control ambiental estará sujeto a la visión de los científicos. Cuatro científicos (dos por Uruguay y dos por Argentina) integrarán un Comité en el seno de la CARU, cuya función será “monitorear el río y todos los establecimientos industriales, agrícolas y centros urbanos que vuelcan sus efluentes al río Uruguay y sus áreas de influencia. La tarea comenzará con la planta de Botnia y la desembocadura del Río". ${ }^{20}$ Los términos del acuerdo aún no logran satisfacer

\footnotetext{
${ }^{20}$ Ver Diario La Capital de Rosario del 29 de julio
} de 2010 las expectativas de la sociedad civil de Gualeguaychú, ya que la pretensión en términos de justicia ambiental radica en que el monitoreo a la planta sea diario y no mensual, característica que adquiriría y que aún está en debate, acerca de las interpretaciones sobre la letra chica del convenio.

Sin duda el eje puesto en la centralidad del rol de los científicos no cierra la polémica, ya que este tema ha sido materia de debate entre los estudiosos de los temas ambientales y particularmente si tenemos en cuenta que estamos hablando de un conflicto de distribución ecológica.

En este sentido es preciso acotar el papel del experto y ubicar el papel del científico técnico, relevando la consulta ciudadana ya que la política ambiental en manos de los expertos se despolitiza y se convierte en gestión (Gudynas,2001:28). Los técnicos y expertos son quienes realizan los ElA, los que deben tener capacidad explicativa, son quienes deben poder comunicarlos en términos comprensibles, tanto para la comunidad como para los tomadores de decisiones. LoS EIA si bien teóricamente apuntan a analizar el uso racional de los recursos naturales, proteger la calidad de vida del medio ambiente y mejorar la calidad de vida de las personas ${ }^{21}$, se han convertido en uno de los principales aportes para la toma de decisiones gubernamentales, en especial para los casos de grandes obras.

Se ha advertido acerca de que los ElA operan como filtro, manipulando la participación de la sociedad civil y limitando sus prácticas (Leis, 1997:238), que pueden presentarse desde la ofensiva retórica sobre el discurso de la sustentabilidad, y de hecho esto ha sucedido, ya que, el alcance de

${ }^{21}$ Según la definición del PNUMA(Programa de las Naciones Unidas para el Desarrollo) 
fiabilidad de los estudios durante dl devenir de este conflicto ha sido relativizado, tanto por el gobierno como por la sociedad en general.

En este sentido, para el aparente inicio de definición de una nueva etapa ya que parecería atinado hablar de resolución - en el conflicto de las pasteras, lo que ahora se está considerando como materia de discusión es cuáles criterios definirán el cuidado de los bienes ambientales en la contaminación del agua, cuáles serán los indicadores que se construirán y de que manera estarán presentes en los ElA. Y aquí entramos nuevamente en un terreno político: los estudios de impacto ambiental no son neutros. Los EIA son análisis y evaluaciones que permiten que los actores sociales puedan disponer de ellos para formar opinión pública, generar debates de comunicación política y por lo tanto aspirar a poder incidir en el sistema político, en las decisiones de política ambiental. Es que el espacio de la opinión pública tiene una función crítica por excelencia, donde se forma influencia y es en él donde los actores luchan por ejercerla.

\section{Referencias Bibliográficas}

ACHKAR, Marcel, DOMínGUEZ, Ana y PESCE, Fernando (2005)."Uruguay y la profundización de un modelo" En: Entre el desierto verde y el país productivo. El modelo forestal en Uruguay y en el Cono Sur. Edición Casa Bertolt Brecht y REDES-Amigos de la Tierra, Montevideo. (pág. 48-57)

-ARAYA DUJISIN, Rodrigo. (2001) “Ecología de la información: introducción"en: Ecología de la información. Escenarios y actores para la participación ciudadana en asuntos. ambientales. , Araya Dujisin Editor. FLACSO CHILE/ Editorial Nueva Sociedad, Venezuela. (pág. 9-18)

BARROS, Adriana (organizadora). Sustentabilidad, diversidad y democracia. Coalición Ríos Vivos. Campo Grande-MS, 2003.

-CARNEIRO. Eder. "Política ambiental e ideología do desenvolvimento sustentable" en: A insustentável leveza da política ambiental. Desenvolvimento e conflictos ambientas. Zhouri, Andrea y otros (organizadores). Editorial Autentica. Belo Horizonte, 2005 (pág.27-48)
-CASTRO, José Esteban (2008) “Los bienes comunes y la ciudadanía: contradicciones de una relación en pleno desarrollo. , en : Genes, bytes y emisiones: bienes comunes y ciudadanía. HELLFRICH, Silke (compiladora). Fundación Heinrich Böll Stiftung . (pág.6880)

-COLACRAI, Miryam (2008)“Las plantas de celulosa sobre el Río Uruguay y el rol de los actores subnacionales gubernamentales en la proyección de la política Exterior del lado argentino. Acciones, tensiones políticas y negociaciones diplomáticas,2003-2007", en : Revista Tema y Debates. Facultad de Ciencia Políticas y Relaciones Internacionales. Universidad Nacional de Rosario. UNR Editora. (pág. 9-34)

-COLOMBO, Marcela Et Al .(2005) “Cuando la comunidad busca hacerse oír. El conflicto de los residuos sólidos urbanos del Gran San Miguel de Tucumán", en: Revista Iberoamericana de Economía Ecológica Vol2. Revista de la Red Iberoamericana de Economía Ecológica(versión electrónica), disponible en: www.redibec.org/archivos/redibec.htm (pág.9-19) 
CUENCA Berger, Lucio (2005). “Celulosa Arauco en Valdivia. El desastre ambiental en el Río Cruces, resultado del modelo forestal chileno". En: Entre el desierto verde y el país productivo. El modelo forestal en Uruguay y en el Cono Sur. Edición Casa Bertolt Brecht y REDES-Amigos de la Tierra, Montevideo. (pág.35-43)

GEARY, Mirta (2004) “Políticas públicas y sociedad civil. Debate sobre cooperación y negociación en cuestiones ambientales". En: Segundas Jornadas de la Asociación Argentino Uruguaya de Economía Ecológica (ASAUEE). Versión electrónica ISBN:98722038-0-6. Compiladores: Horacio FeinsteinWalter Pengue y Pedro Tsakoumagkos. Lujan, Argentina. (Pág.1-15)

(2006) “El conflicto de las papeleras". En: Revista Otro Sur. Facultad de Ciencia Política y Relaciones Internacionales. Rosario. (pág. 30-34)

GUDYNAS, Eduardo. (2001) "Incertidumbre, riesgo y conocimiento experto, implicaciones para las políticas ambientales", en Ecología de la información. Escenarios y actores para la participación ciudadana en asuntos ambientales, Araya Dujisin Editor. FLACSO CHILE/ Editorial Nueva Sociedad, Venezuela, 2001 (pág.17-34)

HABERMAS, Jünger (2000). Facticidad y validez. Editorial Trotta, Madrid, 2000. (pág. 407-468)

LEIS, Héctor Ricardo (2001). La modernidad insustentable. PNUMA- Nordan, Montevideo.(pág.9-222)

LEFF, Enrique (1998). Saber ambiental: sustentabilidad ambiental, racionalidad, complejidad y poder. Siglo Veintiuno Editores/PNUMA. México, 1998. (pág. 15275)
MARTÍNEZ ALIER, Joan (2006) Los conflictos ecológico-distributivos y los indicadores de sustentabilidad. REDALYC. Revista de la Universidad Bolivariana.Chile. ISSN (versión impresa) 0717-6554

MARTÍNEZ ALIER, Joan y otro(2000). Economía ecológica y política ambiental. Fondo de Cultura Económica y PNUMA (Programa de las Naciones Unidas para el Desarrollo).(pág.11-483)

MARTÍNEZ ALIER, Joan. (1997) "Lusticia ambiental" en: Meio Ambiente. Desenvolvimiento sustentable e políticas públicas.Clovis Cavalcanti (org). Cortez Editora- Fundación Joaquín Tabuco, San Pablo, Brasil. (pág.215-231)

MEI RELLES, Daniela. "Forestación en el Cono Sur"(2005) en: Entre el desierto verde y el país productivo.El modelo forestal en Uruguay y en el Cono Sur. Edición Casa Bertolt Brecht y REDES-Amigos de la Tierra, Montevideo.- (pág. 28-34)

MERLINSKY, María Gabriela (2008). “La gramática de la acción colectiva ambiental en Argentina: reflexiones en torno al movimiento ciudadano ambiental de Gualeguaychú y su inscripción en el espacio público", en : Revista Tema y Debates. Facultad de Ciencia Políticas y Relaciones Internacionales. Universidad Nacional de Rosario. UNR Editora. (pág.35-60)

PERUZZOTTI Y SMULOVITZ (2001).

"Accountabilty social: la otra cara del control", en: Controlando la política. Ciudadanos y medios en las nuevas democracias latinoamericanas (Peruzzotti y 
Smulovitz editores) . Editorial Temas,

Buenos Aires, 2001. (pág.23-52)

-RAMOS, Pablo (2006). “Papeleras: 10 claves para entender el costado económico". De la Agencia Periodística del Mercosur (APM). Organo de Producción de la Facultad de Periodismo y Comunicación Social de la UNLP (Universidad NACIONAL DE La Plata). Febrero 2006.

WILLAT, Fernando(2005). “Instalación de plantas de celulosa en Fray Bentos: un enfoque de derechos. En: Entre el desierto verde y el país productivo. El modelo forestal en Uruguay y en el Cono Sur. Edición Casa Bertolt Brecht y REDES-Amigos de la Tierra, Montevideo. (pág.63-71)

WOLTON, Dominique (1995). La comunicación política: construcción de un modelo, , en: "El nuevo espacio público", AAVV, Gedisa, 1995 (pág.28-46)

ZIBECHI, Raúl (2005) “Celulosa y forestación, dos caras de un modelo depredador". En: Entre el desierto verde y el país productivo. El modelo forestal en Uruguay y en el Cono Sur. Edición Casa Bertolt Brecht y REDESAmigos de la Tierra, Montevideo (pág.92-97) 